\title{
Probing the early Universe from deep underground
}

\author{
Francesca Cavanna $^{1, *}$ for the LUNA collaboration ${ }^{2}$ \\ ${ }^{1}$ INFN Sezione di Torino \\ ${ }^{2}$ https://luna.lngs.infn.it
}

\begin{abstract}
Big Bang Nucleosynthesis (BBN) occurs during the first minutes of cosmological time in a rapidly expanding hot and dense Universe, where a fraction of protons and nearly all free neutrons end up bound in ${ }^{4} \mathrm{He}$, while $\mathrm{D}$, ${ }^{3} \mathrm{H},{ }^{3} \mathrm{He},{ }^{6} \mathrm{Li},{ }^{7} \mathrm{Li}$ and ${ }^{7} \mathrm{Be}$ nuclei form in trace quantities. Among these elements, deuterium is an excellent indicator of cosmological parameters because its abundance is highly sensitive to the primordial baryon density and to the number of relativistic species. Although astronomical observations of primordial deuterium abundance have reached percent accuracy, theoretical predictions based on BBN were hampered by large uncertainties on the cross-section of the deuterium burning $\mathrm{D}(\mathrm{p}, \gamma)^{3} \mathrm{He}$ reaction, before the LUNA measurement. In the following, I will report the results of the experimental campaign carried on at LUNA and its cosmological implications.
\end{abstract}

\section{The primordial deuterium abundance: motivation for the measurement of the $\mathrm{D}(\mathrm{p}, \gamma)^{3} \mathrm{He}$ reaction}

The BBN theory is one of the pillars of the picture we nowadays have of the Universe. In particular, for a given baryon density, it gives the primordial abundance of the light elements. Such predictions can then be compared with the results provided by spectroscopic observations of ancient astrophysical objects, like quasars or metal poor stars.

In the current precision era of cosmology, data from observations are characterized by higher uncertainties with respect to BBN predictions, except for deuterium. In particular, the $\mathrm{D} / \mathrm{H}$ ratio is retrieved by astronomical observations with an excellent accuracy of about $1 \%$ [1], whereas the $\mathrm{BBN}$ prediction of $\mathrm{D} / \mathrm{H}$ has an uncertainty of about $2 \%$ [2]. Actually, the relatively large error on the prediction of the $\mathrm{D} / \mathrm{H}$ was dominated by the uncertainty on the cross sections of the nuclear reactions involved in the BBN network to first create and then destroy deuterium. In particular, before the LUNA measurement [3], the main uncertainty came from the $\mathrm{D}(\mathrm{p}, \gamma)^{3} \mathrm{He}$ reaction responsible for deuterium burning.

Several data sets on the $\mathrm{D}(\mathrm{p}, \gamma)^{3} \mathrm{He}$ reaction cross section, or equivalently its $S(E)$-factor were available in the literature before the LUNA measurement. The astrophysical $S(E)$ factor is defined as: $S(E)=E \sigma(E) \exp (2 \pi \eta)$, where $E$ is the energy of interaction in the centre of mass system, $\sigma(E)$ is the energy dependent cross-section, and $\eta$ is the Sommerfeld parameter $\eta(E)=\mathrm{Z}_{1} \mathrm{Z}_{2} \alpha\left(\mu c^{2} / 2 E\right)^{1 / 2}$ (with $\mathrm{Z}_{i}$ atomic numbers of the interacting particles, $\alpha$ fine structure constant, $\mu$ reduced mass, and $c$ speed of light). In the low-energy

\footnotetext{
*e-mail: francesca.cavanna@to.infn.it
} 
range $\left(E_{\text {C.M. }} \simeq 2-20 \mathrm{keV}\right)$, mostly relevant to hydrogen burning in the Sun and in protostars, cross sections were obtained with a systematic error of at most 5.3\% [4] using the $50 \mathrm{kV}$ accelerator (now in disuse) of the Laboratory for Underground Nuclear Astrophysics (LUNA) at the Laboratori Nazionali del Gran Sasso (LNGS) in Italy [5]. At higher energies $\left(E_{\text {C.M. }} \simeq 30-700 \mathrm{keV}\right)$, available data sets were affected by systematic errors of $9 \%$ or higher [6-9]. The situation was further compounded by the fact that a recent ab initio calculation [10] disagrees at the 20-30\% level with both the $S$-factor of Ma et al. [6] and a best fit [11] to selected data $[4,6,12,13]$, widely used in BBN calculations. These large uncertainties had significant impact on the comparison between predicted and observed primordial abundance, which is of crucial importance to access the baryon density of the Universe and to test more exotic scenarios beyond the Standard Model of fundamental interactions. As a consequence of the poor experimental data for the $\mathrm{D}(\mathrm{p}, \gamma)^{3} \mathrm{He}$ reaction, firm conclusions on the BBN stage and cosmological model couldn't be drawn with a satisfactory precision. For all these reasons, a new experimental campaign started at LUNA in 2016.

\section{The $\mathrm{D}(\mathrm{p}, \gamma)^{3} \mathrm{He}$ cross section measurement: experimental setup and data analysis}

The measurements were carried out using the LUNA facility located in the underground Gran Sasso National Laboratory (LNGS), Italy. The LUNA400 accelerator provided a high intensity proton beam (beam current $100-300 \mu \mathrm{A}$ ) in the 50-400 keV energy range. The beam entered in a cylindrical reaction chamber $33 \mathrm{~cm}$ long and with a $56 \mathrm{~mm}$ inner diameter, through a $40 \mathrm{~mm}$ long collimator having a inner diameter of $7 \mathrm{~mm}$. High purity molecular deuterium at a 0.3 mbar pressure was present inside the reaction chamber. The beam was stopped by a copper calorimeter that measured its intensity. A large high-purity germanium detector (HPGe) faced to the reaction chamber at $90^{\circ}$ to the beam axis was used to detect the photons emitted by the $\mathrm{D}(\mathrm{p}, \gamma)^{3} \mathrm{He}$ reaction. Possible gas impurities by in-leaking air were periodically checked by the strong $E_{\mathrm{p}}=278 \mathrm{keV}{ }^{14} \mathrm{~N}(\mathrm{p}, \gamma){ }^{15} \mathrm{O}$ resonance and were always below $0.5 \%$ [14].

The $\mathrm{D}(\mathrm{p}, \gamma)^{3} \mathrm{He}(\mathrm{Q}=5.493 \mathrm{MeV})$ reaction proceeds through direct capture to the ${ }^{3} \mathrm{He}$ ground state with the emission of a single gamma with an energy in the range 5.5 - 5.8 MeV. Since these energies are above the natural radioactivity endpoint, it is possible to fully exploit the suppression of cosmic ray induced background due to the Gran Sasso mountain [5, 15]. The incoming proton can be captured both in a $s$-wave or $p$-wave orbital angular momentum state and the emitted gamma-ray shows a not isotropic angular distribution dominated by the M1 and E1 components. To measure the cross section in the energy range of interest $\left(30 \mathrm{keV}<\mathrm{E}_{c m}<300 \mathrm{keV}\right.$ ) for BBN we explored the full dynamic range of the LUNA-400 accelerator in 30-50 keV energy steps. The main source of beam induced background was due to the ${ }^{19} \mathrm{~F}(\mathrm{p}, \alpha \gamma){ }^{16} \mathrm{O}$ reaction from the interaction of protons with fluorine contaminant usually present on collimators along the gas target and on the calorimeter. This beam-induced background was quantified in dedicated control runs in which inert ${ }^{4} \mathrm{He}$ gas was used instead of deuterium. A sample spectrum taken at $150 \mathrm{keV}$ beam energy is shown in figure 1 .

For an extended gas target setup, the cross section is given by the following expression:

$$
\sigma(E)=\frac{N_{\gamma}}{N_{p} \int_{0}^{L} \rho(z) \cdot \epsilon(z) \cdot W(z) d z}
$$

where $N_{\gamma}$ is the number of counts after background subtraction. The integral extends over the target length, in which $\rho(z)$ represents the gas density profile, $\epsilon(z)$ is the gamma detection 




Figure 1: Typical $\gamma$-ray spectrum obtained with the HPGe detector at $150 \mathrm{keV}$ beam energy: in blue the spectrum measured with $\mathrm{D}_{2}$ gas and for comparison a beam induced background spectrum (grey) acquired with ${ }^{4} \mathrm{He}$ gas. The full-energy, single-escape and double-escape peaks from the $\mathrm{D}(\mathrm{p}, \gamma)^{3} \mathrm{He}$ reaction are clearly visible.

efficiency as a function of position along the beam axis, $W(z)$ accounts for the photon angular distribution and $N_{p}$ is the number of projectiles impinging the target.

A major effort was devoted to prevent or minimize any source of systematic uncertainty in equation 1 to reach an accuracy of three percent on the S-factor. $N_{\gamma}$ is essentially affected by the statistical error, that in our measurements was always lower than $1 \%$ for $E_{\mathrm{p}>100} \mathrm{keV}$ $\left(E_{\text {C.M. }}>70 \mathrm{keV}\right)$, as a consequence of the suppression in the cosmic ray induced background with respect to surface experiments. The target density $\rho(z)$ was determined by measuring the pressure and temperature profiles along the target as well as the beam heating effect due to the power released by the incident beam in the gas target [16]. The integrated beam current $N_{p}$ was measured with a consolidated technique in which the beam power was monitored during each run by measuring the power dissipated by the beam on the copper calorimeter where the beam was stopped [17]. In an extended gas target the interaction with proton beam can take place at different positions along the beam axis, resulting in different energies of the emitted photons and in different geonetrical angles subtended by the HPGe detector. Therefore, the $\gamma$-ray detection efficiency $\epsilon(\mathrm{z})$ must be carefully determined as a function of both position and energy. The $\gamma$-rays emitted by the $\mathrm{D}(\mathrm{p}, \gamma)^{3} \mathrm{He}$ reaction $(Q=5.5 \mathrm{MeV})$ have typical energies $E_{\gamma}=5.5-5.8 \mathrm{MeV}$, i.e. far away from the energy of the commonly used radioactive sources. The efficiency $\epsilon(z)$ of the HPGe detector was measured along the beam line, by filling the reaction chamber with $N_{2}$ and exploiting the resonance of the $\left.{ }^{14} N(p, \gamma)\right)^{15} O$ reaction at $E_{\mathrm{r}}=$ $259 \mathrm{keV}$. This reaction produces cascade $\gamma$-rays in a large energy range $(0.7-7 \mathrm{MeV})$, in such a way the efficiency were precisely determined [14]. Finally, the $W(z)$ function has 


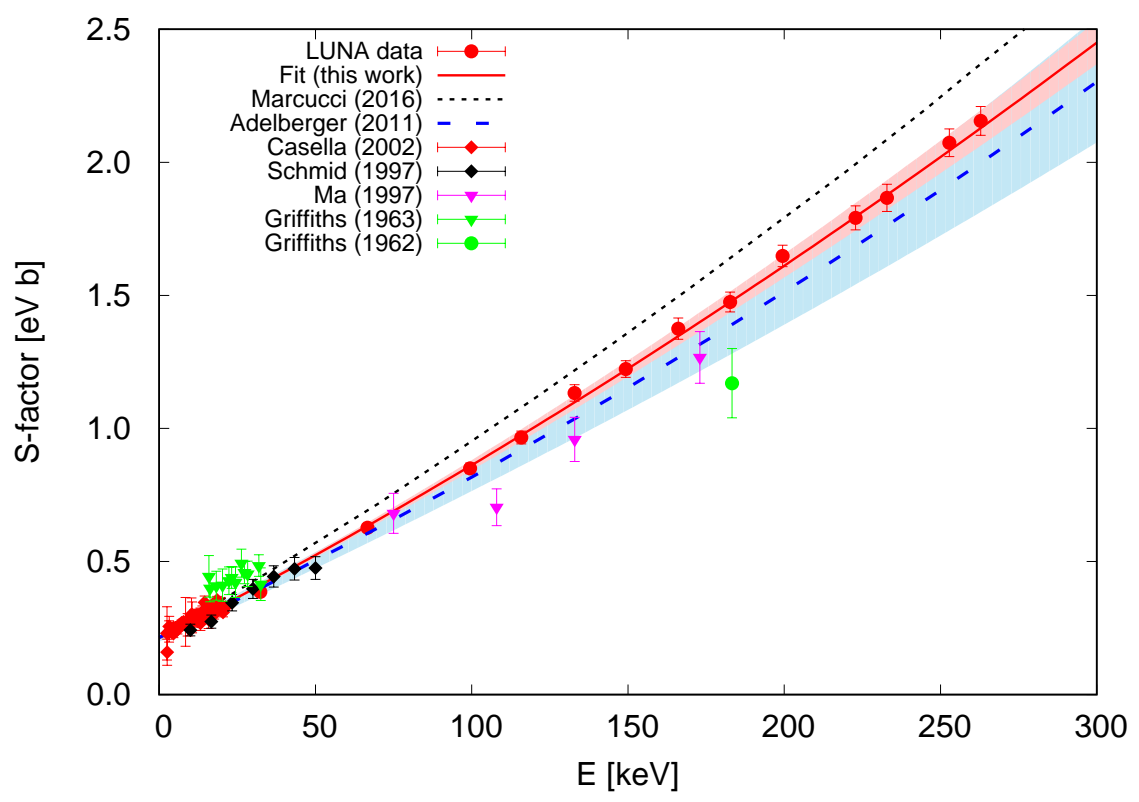

Figure 2: S-factor of the $\mathrm{D}(\mathrm{p}, \gamma)^{3} \mathrm{He}$ reaction; LUNA results are represented with filled red circles. Other experimental data are also shown. The best fit (red solid line) include all the reported experimental data. Band represents the $68 \%$ confidence level.

been unfolded from the shape of full energy peak of photons produced by the $\mathrm{D}(\mathrm{p}, \gamma)^{3} \mathrm{He}$ that depends on the angular distribution of emitted photons. The detailed description of the measurements performed to determine the above mentioned quantities and uncertainties can be found in [14].

After four years of data taking and a long data analysis campaign, it was possible to obtain the S-factor shown in Figure 2 where the LUNA data [3] are reported together with literature data. The new data show a significantly reduced uncertainty with respect to previous experimental dataset. Also shown is the polynomial best fit of $\mathrm{S}(\mathrm{E})$ for $E \sim 0-2 \mathrm{MeV}$ to previous dataset [11] (blue line) and the fit in which this new data set is considered (red line). The bands represent the quoted $1 \sigma$ uncertainty of the best fit values.

\section{Results and conclusions}

To explore the impact of our $\mathrm{D}(\mathrm{p}, \gamma)^{3} \mathrm{He} S$-factor on the predicted primordial deuterium abundance, we used the second release [18] of the numerical BBN code PArthENoPE. Under the assumption of the $\Lambda$ CDM model, with $N_{\text {eff }}=3.045$, we performed a Bayesian likelihood analysis to derive $\Omega_{\mathrm{b}} h^{2}$ using the observed deuterium abundance, $(\mathrm{D} / \mathrm{H})_{\mathrm{obs}}$, and the theoretical behaviour of $(\mathrm{D} / \mathrm{H})_{\mathrm{BBN}}$ (now including the new LUNA data). We obtained $\Omega_{\mathrm{b}} h^{2}(\mathrm{BBN})=0.02233 \pm 0.00036$, a value which is a factor of 2 more precise than that de- 
duced using a previous $S$ factor [11] and now in much better agreement with the $\Omega_{\mathrm{b}} h^{2}$ based on CMB data [19].

If we use the baryon density provided by the PLANCK Collaboration [20], we derive a theoretical prediction on deuterium abundance $(\mathrm{D} / \mathrm{H})_{\mathrm{BBN}}=(2.52 \pm 0.03 \pm 0.06) \times 10^{-5}$, in excellent agreement with astronomical observations $[1](\mathrm{D} / \mathrm{H})_{\mathrm{obs}}=(2.527 \pm 0.030) \times 10^{-5}$.

After the release of the LUNA results [3], several papers were published discussing the cosmological implications with the inclusion of the new $\mathrm{D}(\mathrm{p}, \gamma)^{3} \mathrm{He}$ rate [2, 21-23]. All the authors agree on the need of new measurements of the $\mathrm{D}(\mathrm{d}, \mathrm{n})^{3} \mathrm{He}$ and $\mathrm{D}(\mathrm{d}, \mathrm{p})^{3} \mathrm{H}$ reactions which have became the most prominent source of uncertainty in the determination of the primordial deuterium abundance.

\section{References}

[1] R.J. Cooke, M. Pettini, C.C. Steidel, Astrophys. J. 855, 102 (2018), 1710.11129

[2] O. Pisanti, G. Mangano, G. Miele, P. Mazzella, JCAP 04, 020 (2021), 2011.11537

[3] V. Mossa, K. Stöckel, F. Cavanna, F. Ferraro, M. Aliotta, F. Barile, D. Bemmerer, A. Best, A. Boeltzig, C. Broggini et al., Nature 587, 210 (2020)

[4] C. Casella, H. Costantini, A. Lemut, B. Limata, R. Bonetti, C. Broggini, L. Campajola, P. Corvisiero, J. Cruz, A. D’Onofrio et al., Nucl. Phys. A 706, 203 (2002)

[5] F. Cavanna,, P. Prati, Int. J. Mod. Phys. A 33, 1843010-346 (2018)

[6] L. Ma, H. Karwowski, C. Brune, Z. Ayer, T. Black, J. Blackmon, E. Ludwig, M. Viviani, A. Kievsky, R. Schiavilla, Phys. Rev. C 55, 588 (1997)

[7] G. Schmid, B. Rice, R. Chasteler, M. Godwin, G. Kiang, L. Kiang, C. Laymon, R. Prior, D. Tilley, H. Weller, Phys. Rev. C 56, 2565 (1997)

[8] G. Griffiths, M. Lal, C. Scarfe, Can. J. Phys. 41, 724 (1963)

[9] I. Tišma, M. Lipoglavšek, M. Mihovilovič, S. Markelj, M. Vencelj, J. Vesić, Eur. Phys. J. A 55, 137 (2019)

[10] L. Marcucci, G. Mangano, A. Kievsky, M. Viviani, Phys. Rev. Lett. 116, 102501 (2016)

[11] E. Adelberger, A. García, R. Robertson, K. Snover, A. Balantekin, K. Heeger, M. Ramsey-Musolf, D. Bemmerer, A. Junghans, C. Bertulani et al., Rev. Mod. Phys. 83, 195 (2011)

[12] G. Griffiths, E. Larson, L. Robertson, Can. J. Phys. 40, 402 (1962)

[13] G. Schmid, M. Viviani, B. Rice, R. Chasteler, M. Godwin, K. G.C., L. Kiang, A. Kievsky, C. Laymon, R. Prior et al., Phys. Rev. Lett. 76, 3088 (1996)

[14] V. Mossa, K. Stöckel, F. Cavanna, F. Ferraro, M. Aliotta, F. Barile, D. Bemmerer, A. Best, A. Boeltzig, C. Broggini et al., Eur. Phys. J. A 56, 144 (2020), 2005.00002

[15] F. Ferraro, G.F. Ciani, A. Boeltzig, F. Cavanna, S. Zavatarelli, 7, 119 (2021)

[16] F. Cavanna, R. Depalo, M.L. Menzel, M. Aliotta, M. Anders, D. Bemmerer, C. Broggini, C.G. Bruno, A. Caciolli, P. Corvisiero et al., 50, 179 (2014), 1411.2888

[17] F. Ferraro, M. Takács, D. Piatti, V. Mossa, M. Aliotta, D. Bemmerer, A. Best, A. Boeltzig, C. Broggini, C. Bruno et al., Eur. Phys. J. A 54, 44 (2018)

[18] R. Consiglio, P. de Salas, G. Mangano, G. Miele, S. Pastor, O. Pisanti, Comp. Phys. Comm. 233, 237 (2018)

[19] B.D. Fields, K.A. Olive, T.H. Yeh, C. Young, Journ. Cosm. and Astropart. Phys. 2020, $010(2020)$

[20] N. Aghanim, Y. Akrami, M. Ashdown, J. Aumont, C. Baccigalupi, M. Ballardini, A. Banday, R. Barreiro, N. Bartolo, S. Basak et al., arXiv e-prints arXiv:1807.06209 (2018) 
[21] T.H. Yeh, K.A. Olive, B.D. Fields, JCAP 03, 046 (2021), 2011. 13874

[22] C. Pitrou, A. Coc, J.P. Uzan, E. Vangioni, Mon. Not. Roy. Astron. Soc. 502, 2474 (2021), 2011.11320

[23] J. Moscoso, R.S. de Souza, A. Coc, C. Iliadis, Astrophys. J. 923, 49 (2021) 